\title{
A Centroid Model for the Depth Assessment of Images using Rough Fuzzy Set Techniques
}

\author{
P. Swarnalatha and B.K. Tripathy \\ School of Computing Sciences and Engineering, \\ VIT University, Vellore 632014, TN, INDIA \\ Email: pswarnalatha@vit.ac.in and tripathybk@vit.ac.in
}

\begin{abstract}
Detection of affected areas in images is a crucial step in assessing the depth of the affected area for municipal operators. These affected areas in the underground images, which are line images are indicative of the condition of buried infrastructures like sewers and water mains. These images identify affected areas and extract their properties like structures from the images, whose contrast has been enhanced. A Centroid Model for the Depth Assessment of Images using Rough Fuzzy Set Techniques presents a three step method which is a simple, robust and efficient one to detect affected areas in the underground concrete images. The proposed methodology is to use segmentation and feature extraction using structural elements. The main objective for using this model is to find the dimensions of the affected areas such as the length, width, depth and the type of the defects/affected areas. Although human eye is extremely effective at recognition and classification, it is not suitable for assessing defects in images, which might have spread over thousands of miles of image lines. The reasons are mainly fatigue, subjectivity and cost. Our objective is to reduce the effort and the labour of a person in detecting the affected areas in underground images. A proposal to apply rough fuzzy set theory to compute the lower and upper approximations of the affected area of the image is made in this paper. In this connection we propose to use some concepts and technology developed by Pal and Maji.
\end{abstract}

Index Terms - Histogram Equalization, Contrast Stretching, Image Enhancement, Image Segmentation and Feature Extraction, Rough Fuzzy Set Techniques.

\section{INTRODUCTION}

Affected areas of buried infrastructure facilities such as sewer images pose a serious problem to most developed urban centres today [8]. They may deteriorate and ultimately fail to fulfill their intended functions. To ensure continuity of services and protect the investment made in these networks, municipalities check their conditions regularly. Condition assessment is done by using CCTV (Closed Circuit Television) or SSET (Sewer Scanner Evaluation Technology) cameras mounted on robots producing a video record or a digital image of the image's condition. The video records are later evaluated by a human operator and affected areas are classified against documented criteria. Factors like experience of the operator, skill level, concentration and reliability of the picture quality significantly influence the accuracy of affected area or failure diagnosis. Although image scan data is acquired using automated technologies like the CCTV or SSET, defect detection and classification is still done by manual operators on the field or off-line.

\subsection{Problems Involved}

The defect detection by human eye brings in the issue of subjectivity, difficulty in classification, fatigue and life-cycle cost over extended periods. Therefore, reliable automated affected area detection and classification systems are highly desirable to compensate for the subjective interpretation of the actual condition of buried sewer images.

\subsection{Solution to the above problem}

An automated analysis of scanned underground image images using Image Processing can be used to overcome the above mentioned problem. Instead of manual inspection, here the system processes the obtained input images and identifies the affected area and its other parameters.

It involves:

- Recognition of individual objects and their analysis (partitioning of the image into regions or objects).

- "Making sense" of an ensemble of individual recognized objects (extracting the desired features).

Fuzzy set theory and rough set theory [11] are to models to take care of impreciseness in data. Since our problems also involve impreciseness in the form of different measures we need some such model and analysis based upon them to achieve higher precision. From the outset these theories appear to be competing models. However as has been established by Dubois and Prade [1], they complement each other. Also, it has been found in many real life applications that hybrid models featuring both these models like fuzzy rough sets or rough fuzzy sets are better models than the individual ones. So, here we propose to use the rough fuzzy model foe representation and analysis of our problems.

Hence, integration of probabilistic and possibilistic membership functions may avoid the problems of noise sensitivity of the FCM (fuzzy c-means) and the coincident clusters of the PCM (possibilistic c-means). Each partition is represented by a set of three parameters, namely, a cluster prototype (centroid), a crisp lower approximation, and a fuzzy boundary. The lower 
approximation influencing the fuzziness of the final partition may be obtained [7].

\section{METHODOLOGY}

This system works in an efficient manner. The images of the underground images are taken as an input from the user and using various image enhancement techniques such as histogram equalization, contrast stretching, noise removal and spatial averaging the contrast of the image is improved and an enhanced image is obtained. To this improved image various segmentation techniques such as thresholding and edge detection are applied and the pixels in the image are classified into one of the two classes: background and affected area. The defects can be extracted from the image containing it using structuring elements and cropping the defect/affected area. For the defect thus obtained its dimensions (i.e. length and breadth) are calculated. The user is expected to give certain parameters as an input. Using the parameters obtained and the available dimensions the depth of the image can be estimated. Applying a threshold on the length and width obtained the type of defect can be determined. The types of defects are classified into two classes such as: crack and hole. The affected area of an image can also be estimated, based on which it can be concluded whether the image can be mended or whether it has to be replaced with a new one.

This system helps in finding out the exact location of the defects underground and also helps in estimating their dimensions along with the type of defects more accurately without encountering the above mentioned problems.

His system follows image and filter architectural style. This style is proposed due to the reason that output of one component or module is taken as an input for the next component or module. The processing does not materialize if any of the modules is skipped or the processing is not done in a proper flow. Hence, a proper sequence must be followed. The diagram represented below describes the division of the system, the processes performed by it and the flow of information across the modules of the system. And the new centroid may be calculated based on the weighting average of the crisp lower approximation and fuzzy boundary for better results [7].

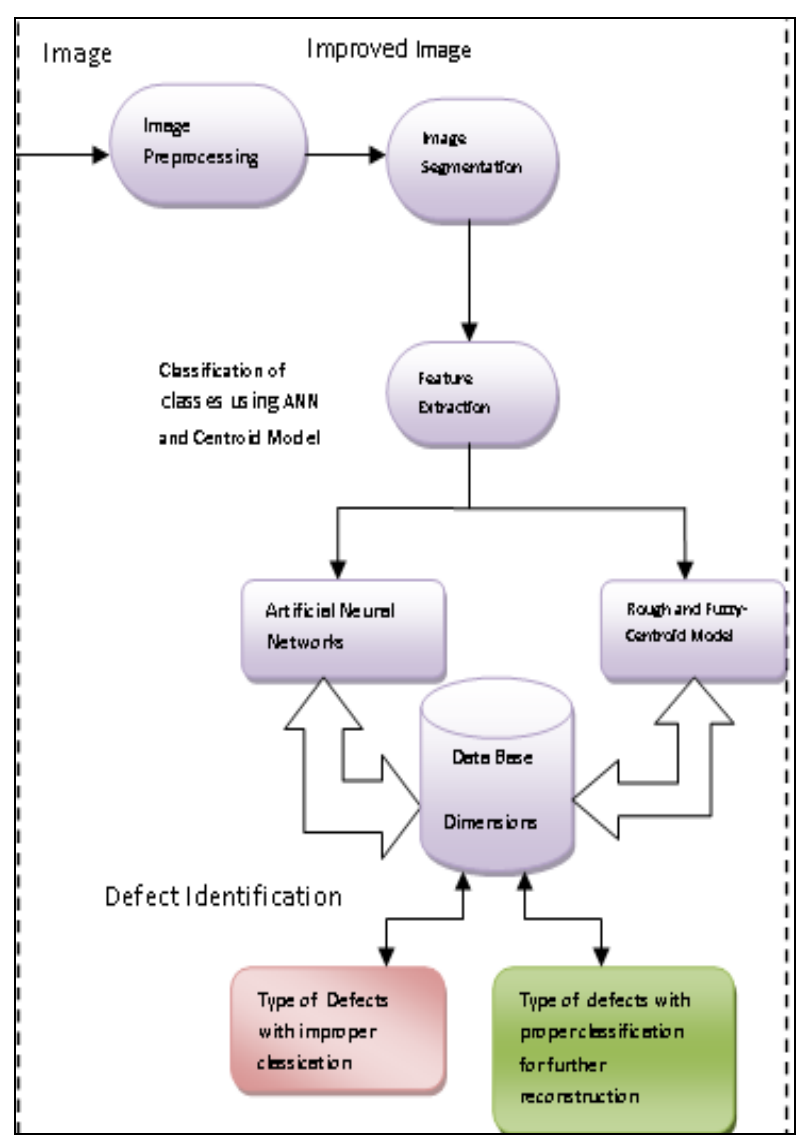

Fig. 1:Block diagram of a centroid model for depth assessment of images using rough fuzzy set techniques

\subsection{Definition of Rough Sets:}

The notion of rough sets was introduced by Pawlak [6] in the year 1982 as an extension of the crisp sets. We provide below the definition of a rough set.

Let $U(\neq \varnothing)$ be a finite set of objects, called the universe and $\mathrm{R}$ be an equivalence relation over $\mathrm{U}$. By $U / R$ we denote the family of all equivalence classes of $R$ (or classification of $\mathrm{U}$ ) referred to as categories or concepts of $\mathrm{R}$ and $[\mathrm{x}]_{\mathrm{R}}$ denotes a category in $\mathrm{R}$ containing an element $x \in U$. By a Knowledge base, we understand a relation system $k=(U, R)$, where $U$ is as above and $R$ is a family of equivalence relations over $\mathrm{U}$.

For any subset $\mathrm{P}(\neq \varnothing) \subseteq \mathrm{R}$, the intersection of all equivalence relations in $\mathrm{P}$ is denoted by IND $(\mathrm{P})$ and is called the indiscernibility relation over $P$. The equivalence classes of IND (P) are called P- basic knowledge about $\mathrm{U}$ in $\mathrm{K}$. For any $\mathrm{Q} \in \mathrm{R}$, $\mathrm{Q}$ is called a $\mathrm{Q}-$ elementary knowledge about $U$ in $K$ and equivalence classes of $\mathrm{Q}$ are called Q-elementary concepts of knowledge R. The family of P-basic categories for all $\varnothing$ $\neq \mathrm{P} \subseteq \mathrm{R}$ will be called the family of basic categories in knowledge base K. By IND (K), we denote the family of 
all equivalence relations defined in $\mathrm{k}$. Symbolically, IND $(\mathrm{K})=\{\mathrm{IND}(\mathrm{P}): \varnothing \neq \mathrm{P} \subseteq \mathrm{R}\}$.

For any $\mathrm{X} \subseteq \mathrm{U}$ and an equivalence relation $\mathrm{R} \in \mathrm{IND}(\mathrm{K})$, we associate two subsets, $R X=\bigcup\{Y \in U / R: Y \subseteq X\} \quad$ and $\overline{\bar{R}} X=\bigcup\{Y \in U / R: Y \cap X \neq \varnothing\}$, called the R-lower and $\mathrm{R}$-upper approximations of $\mathrm{X}$ respectively. The $\mathrm{R}$ boundary of $\mathrm{X}$ is denoted by $\mathrm{BN}_{\mathrm{R}}(\mathrm{X})$ and is given by $\mathrm{BN}_{\mathrm{R}}(\mathrm{X})=\bar{R} X-\underline{R} X$. The elements of $\underline{R} X$ are those elements of $U$ which can be certainly classified as elements of X employing knowledge of $\mathrm{R}$. The borderline region is the undecidable area of the universe. We say $\mathrm{X}$ is rough with respect to $\mathrm{R}$ if and only if $\underline{R} X \neq \bar{R} X$, equivalently $\mathrm{BN}_{\mathrm{R}}(\mathrm{X}) \neq \varnothing . \mathrm{X}$ is said to be $\mathrm{R}$ - definable if and only if $\underline{R} X=\bar{R} X$, or $\mathrm{BN}_{\mathrm{R}}(\mathrm{X})=\varnothing$. So, a set is rough with respect to $\mathrm{R}$ if and only if it is not $\mathrm{R}$-definable.

\subsection{Definition of Fuzzy Sets:}

In his seminal paper published in 1965 [2], Lotfi A. Zadeh introduced the concept of Fuzzy sets, which uses the concept of graded membership of elements instead of dichotomous membership of crisp sets. Next, we define fuzzy sets below.

The most commonly used range of values of membership functions is the unit interval $[0,1]$. We shall denote the membership function of a fuzzy set $\mathrm{A}$ by $\mu_{A}$, which is defined as $\mu_{A}: U \rightarrow[0,1]$, such that for each $x \in U, \mu_{A}(x)=\alpha, 0 \leq \alpha \leq 1$.

The membership function assigns values to elements in a specified range. This value indicates the grade of membership of the elements. The higher membership value indicates greater degree of membership.

Each fuzzy set is completely and uniquely defined by one particular membership function. The fuzzy membership functions are not only dependent upon the concept but also upon the context.

\subsection{Rough Fuzzy Sets:}

In the beginning when rough sets were introduced by Pawlak in the early 1980 s, it was supposed to be a rival to the theory of fuzzy sets. But it was established by Dubois and Prade [1] that instead of being rival theories, they complement each other. In fact they combined these two models to develop the hybrid models of fuzzy rough sets and rough fuzzy sets.

The notion of rough fuzzy sets was introduced by Dubois and Prade [1] as follows.

Definition 2.3.1: Let (U, R) be an approximation space and $\mathrm{U} / \mathrm{R}=\left\{X_{1}, X_{2}, \ldots X_{n}\right\}$. Then for any $\mathrm{X} \in \mathrm{F}(\mathrm{U})$,

$\underline{R} X$ and $\bar{R} X$, the lower and upper approximations of $\mathrm{X}$ with respect to $\mathrm{R}$ are fuzzy sets in $\mathrm{U} / \mathrm{R}$. That is, $\underline{R} X, \bar{R} X: U / R \rightarrow[0,1]$, such that

(2.3.1) $(\underline{\mathrm{RX}})\left(\mathrm{X}_{j}\right)=\inf _{\mathrm{y} \in X_{j}} \mathrm{X}(\mathrm{y})$. and

$$
(\bar{R} X)\left(X_{j}\right)=\sup _{y \in X} X(y), \text { for all } j=1,2, \ldots n .
$$

The pair $(\underline{R X}, \bar{R} X)$ is called a rough fuzzy set associated with $X$

\section{DETECTION OF DEFECTS/AFFECTED AREAS}

The input is the image of an underground image. First of all, the defect should be highlighted when compared to the background in order to group them easily into different classes. So, Image Enhancement techniques are used and pre-processing is done on the images.
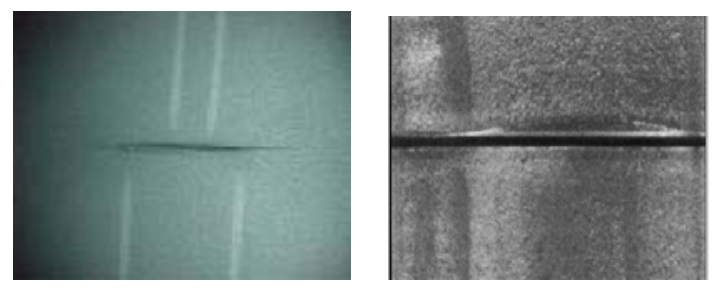

Fig 2: Images of a cracked image

\subsection{Image Enhancement}

Enhancement techniques are used to bring out the detail that is obscured or certain features of interest are highlighted in an image. An example of enhancement is when the contrast of the image is increased to make the image look better. There are different techniques that can be used for image enhancement. They are as follows: histogram equalization, contrast stretching and spatial averaging.

\subsubsection{Histogram Equalization}

Let the variable $r$ represent the gray levels of the image to be enhanced. We assume that $r$ has been normalised to the interval $[0,1]$, with $\mathrm{r}=0$ representing black and $r=1$ representing white. Later, we consider a discrete formulation and allow pixel values to be in the interval $[0, \mathrm{~L}-1]$ where $\mathrm{L}$ is the highest gray level value.

For any $r$ satisfying the aforementioned conditions, we focus attention on transformations of the form

$$
\mathrm{s}=\mathrm{T}(\mathrm{r}) \quad 0 \leq \mathrm{r} \leq 1
$$

that produces a level $\mathrm{s}$ for every pixel value $\mathrm{r}$ in the original image. We assume that the transformation function $\mathrm{T}(\mathrm{r})$ satisfies the following conditions:

(a) $\mathrm{T}(\mathrm{r})$ is single - valued and monotonically increasing in the interval $0 \leq \mathrm{r} \leq 1$; and

(b) $0 \leq \mathrm{T}(\mathrm{r}) \leq 1$ for $0 \leq \mathrm{r} \leq 1$.

Let $p_{r}(r)$ and $p_{s}(s)$ denote the probability density functions of random variables $r$ and $s$, respectively, where the subscripts on $p$ are used to denote that $p_{r}$ and $p_{s}$ are different

functions. 
For discrete values we deal with probabilities and summations instead of probability density functions and integrals. The probability of occurrence of gray level $r_{k}$ in an image is approximated by

$$
\mathrm{P}_{\mathrm{r}}\left(\mathrm{r}_{\mathrm{k}}\right)=\mathrm{n}_{\mathrm{k}} / \mathrm{n} \quad \mathrm{k}=0,1,2, \ldots, \mathrm{L}-1
$$

Where, $\mathrm{n}$ is the total number of pixels in the image, $\mathrm{n}_{\mathrm{k}}$ is the number of pixels that have gray level $r_{k}$, and $L$ is the total number of possible gray levels in the image. The discrete version of the transformation function given in Eq. (1.2) is

$$
\begin{aligned}
& \mathrm{s}_{\mathrm{k}}=\mathrm{T}\left(\mathrm{r}_{\mathrm{k}}\right)=\sum \mathrm{p}_{\mathrm{r}}\left(\mathrm{r}_{\mathrm{j}}\right) \\
& =\sum \mathrm{n}_{\mathrm{j}} / \mathrm{n} \quad \mathrm{k}=0,1,2, \ldots, \mathrm{L}-1 .
\end{aligned}
$$

Thus, a processed (output) image is obtained by mapping each pixel with level $r_{k}$ in the input image into a corresponding pixel with level $s_{k}$ in the output image via Eq. (1.3). A plot of $p_{r}\left(r_{k}\right)$ versus $r_{k}$ is called histogram equalization or histogram linearization.

\subsubsection{Contrast Stretching}

One of the simplest piecewise linear function is contrast-stretching transformation. Low-contrast images can result from illumination, lack of dynamic range in the imaging sensor, or even wrong setting of a lens aperture during image acquisition. The idea behind contrast stretching is to increase the dynamic range of the gray level in the image being processed.

\subsubsection{Spatial Averaging}

The strategy followed in spatial averaging is to utilize the Laplacian to highlight fine detail, and the gradient to enhance prominent edges. The dynamic range of the gray levels is increased by using a gray level transformation.

\subsection{Image Segmentation}

In underground image segmentation, the following classes are of great interest: the image joints (horizontal dark straight lines), image laterals (circular dark objects), surface cracks (irregularly shaped thin dark lines), and the image background (anywhere from a smooth to a highly patterned surface). These can be segmented based on the geometric differences between them, specifically based on morphology. These objects can be distinguished based on their shapes. The shapes being thin and wide (joints), large and round (laterals) and small and irregular (cracks, holes). The horizontal and circular structuring elements can be used for separating them into classes[10][9].

\subsubsection{Edge Detection}

An edge is a set of connected pixels that lie on the boundary between two regions. Edge detection [3] requires the ability to measure gray-level transitions in a meaningful way. Here, this is accomplished by filtering the image initially and then applying suitable mask to the resulting image. Convolution is performed to the above mentioned mask and a $5 \times 5$ matrix and the connected components in the binary image are labeled. The number of connected objects is taken as 8 by default. The pixels labeled 0 are the background. The pixels labeled 1 make up one object; the pixels labeled 2 make up a second object, and so on. The connected pixels with the same label are obtained. This is how an image is divided into segments.

\subsubsection{Thresholding}

Thresholding is one more technique where a particular intensity value is set as a threshold, the initial estimate may be the midpoint between the minimum and maximum intensity values in the image. The midpoint of the above calculated mid points is found out. Segmenting the image using this method will produce two groups. This way segmenting can be done by using thresholding.

Structuring elements are applied to the pixels of the image. That is, using the structuring elements the pixels in the image can be classified into different classes and then by performing the set difference operation the features of the affected area can be extracted from the image for which the horizontal structuring element must be varied. A particular intensity value is considered and all the pixels whose intensity values lie below that value are obtained. Using this technique the affected areas can be obtained.

\subsection{Feature Extraction}

The image is classified into different classes. Now, these features have to be extracted which is done by cropping them. Then, the dimensions of the defects have to be found out. After which the type of defect is determined and also the affected area of an image is estimated.

\subsection{Dimensions of the affected area}

There are several dimensions of the affected area like; length, width, depth and so on. We present these defects/affected areas below.

\subsubsection{Length of the affected area}

The intensities of the pixels are taken into account and column wise calculation is done. The pixels whose intensity values are zero or near to zero which generally represent a dark area, that is, the affected area, are considered. The difference between the maximum and the minimum column values gives us the length of the affected area.

\subsubsection{Width of the defect/affected area}

The intensities of the pixels are taken into account and row wise calculation is done. The pixels whose 
intensity values are zero or near to zero which generally represent a dark area, that is, the affected area, are considered. The difference between the maximum and the minimum row values gives us the width of the defect.

\subsection{Type of defect/affected area}

A threshold value is applied to the length and width of the defect and based on the defect characteristics it is classified into one of the two classes: crack and hole. If the values of length and width are exactly equal or with a difference of $\pm 2 \mathrm{~mm}$ then it is a hole and the affected areas which don't satisfy this condition are cracks.

\subsection{Degree of Affected Area of an Image}

Based on the threshold applied to the length and width of the defect the defects are classified into major defect or minor defect. A defect is said to be a major affected area if the image is deteriorated to a maximum extent. The images with major defects are totally damaged and they have to be replaced and the ones with minor defects can be mended. The condition of the images underground can be estimated using this model.

\subsection{Depth of the defect}

The calculation of depth of the defect [8] is very important as the degree to which the image has been deteriorated can be estimated from this. The angle of incidence of X-rays and the thickness of the image are taken as an input from the user. The figure given below shows the image with defect. The depth of the image can be calculated using the formula given below.
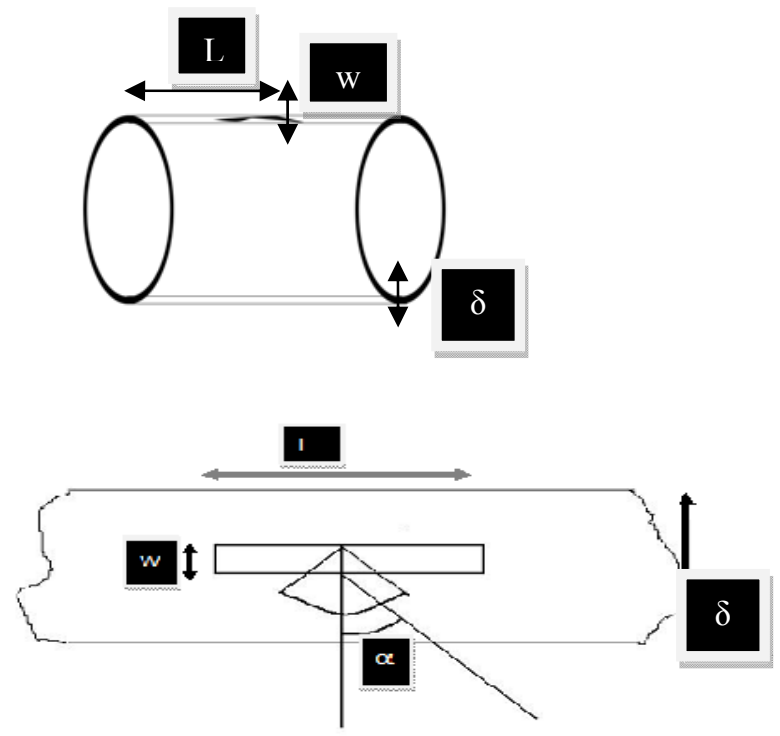

Fig. 4: Depth of the defect
The formula for calculating depth is:

$$
\text { Depth }=\left(\frac{L / 2+x}{\operatorname{Tan} \alpha}+\delta\right)-\frac{W}{\operatorname{Sin} \alpha}
$$

$\mathrm{L}=$ Length of the defect

$\mathrm{x}=0$; Assuming that the defect is exactly in the centre

$\alpha=$ Angle of incidence of the X-rays

$\mathrm{W}=$ Width of the defect

$\delta=$ Thickness of the image

If the depth of the defect is greater than the thickness of the image then the image is fully damaged and it has to be replaced with another one[9].

\subsection{Centroid Model}

The centroid may be calculated based on the weighting average of the crisp lower approximation and fuzzy boundary. Computation of the centroid can be modified to include the effects of both fuzzy memberships (probabilistic and possibilistic) and lower and upper bounds [7]. In this paper, a generalized hybrid algorithm, termed as rough-fuzzy PCM (RFPCM) is proposed, based on rough and fuzzy sets. While the membership function of the fuzzy sets enables efficient handling of overlapping partitions, the concept of lower and upper approximations of rough sets deals with uncertainty, vagueness, and incompleteness in class definition. The algorithm attempts to exploit the benefits of both probabilistic and possibilistic membership functions. Integration of probabilistic and possibilistic membership functions avoids the problems of noise sensitivity of the FCM and the coincident clusters of the PCM. Each partition is represented by a set of three parameters, namely, a cluster prototype (centroid), a crisp lower approximation, and a fuzzy boundary. The lower approximation influences the fuzziness of the final partition. The cluster prototype depends on the weighting average of the crisp lower approximation and fuzzy boundary. The algorithm is generalized in a sense that all existing variants of $c$-means can be derived from the proposed algorithm as a special case. They have derived several quantitative measures based on rough sets to evaluate the performance of the proposed algorithm. It has been concluded that the effectiveness of the new algorithm is higher than the other algorithms in this direction. They have shown how this algorithm can be effectively used for MR image clustering. We can use the same technique to apply the efficient algorithm to our images for finding the affected areas and derive our conclusion to either mend the affected area or to replace the object as a whole.

\section{EXPERIMENTAL RESULTS}

The input to the proposed system is an image which is converted into a gray scale image. To the converted image enhancement techniques are applied in 
order to improve the contrast and sharpness of the image. There are various enhancement techniques out of which one is the histogram equalization which is shown in the figure below
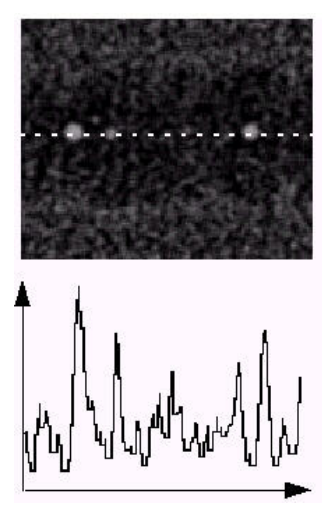
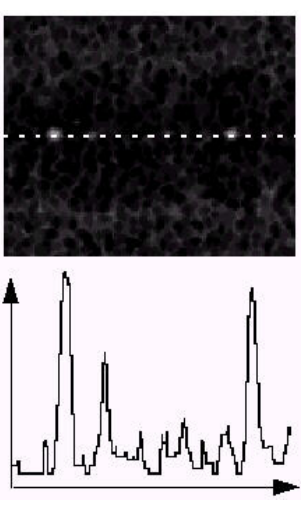

Fig. 5. Histogram of equalized images

The affected area is segmented by the method of global thresholding and then extracted by cropping the desired part. The dimensions of the affected area such as the length, width and depth are calculated. The type of defect is found and then affected areas are classified into major defects and minor defects.
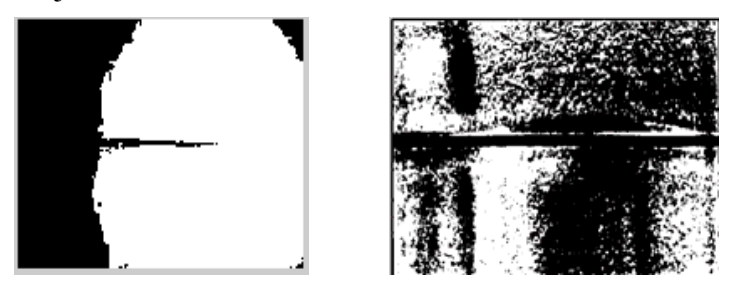

Fig. 6 : Identification of Defect

The Table1 given below shows the images of underground concrete image with affected areas. These images undergo the processing and the proper defect devoid of the background is obtained. The images of different types of image defects are shown in the table. To these defects various techniques are applied and the dimensions are calculated (i.e., the length, width and depth). The thickness of the image and angle of incidence of X-rays are taken as an input from the user and using the formula of depth which has been mentioned above the depth is calculated. To the affected area images we have considered the thickness of the image we are taking as 10 $\mathrm{mm}$ and the angle of incidence of X-rays as $\mathrm{pi} / 2\left(90^{\circ}\right)$. If the depth of the defect thus calculated is more than 10 $\mathrm{mm}$ then the image is said to be fully deteriorated and it has to be replaced with a new one. After calculating the dimensions of the affected area, a threshold is applied to the dimensions based on which the affected area is classified into a crack or a hole. If the length and width of the defect are equal or approximately equal then it is said to be a hole. Later, the affected area is found out by applying another threshold to the dimensions. The defects are classified as major defect or minor defect based on the intensity. If it is a major defect then the image has to be replaced and if it is a minor defect the image can be mended. In the Tablel five different types of defects are considered and all the different attributes are found out based on which the exact position of the defect underground and its dimensions can be estimated.

Table 1: Parameters of Third Dimension

\begin{tabular}{|c|c|c|c|c|}
\hline $\begin{array}{l}\text { Original/En } \\
\text { hanced } \\
\text { Image }\end{array}$ & & & & \\
\hline $\begin{array}{l}\text { Extracted } \\
\text { feature }\end{array}$ & & & & \\
\hline 1D (in mm) & 113 & 11.25 & 222.5 & 31 \\
\hline 2D (in mm) & 3 & 0.5 & 43.25 & 2.25 \\
\hline 3D(inmm) & 6.9998 & 9.5 & $>$ thickness & 7.7499 \\
\hline $\begin{array}{l}\text { Category of } \\
\text { defect }\end{array}$ & Crack & Crack & Crack & Crack \\
\hline $\begin{array}{l}\text { Assessment } \\
\text { of Defect }\end{array}$ & $\begin{array}{l}\text { Major } \\
\text { defect }\end{array}$ & $\begin{array}{l}\text { Minor } \\
\text { defect }\end{array}$ & Major defect & $\begin{array}{l}\text { Major } \\
\text { defect }\end{array}$ \\
\hline
\end{tabular}

\section{CONCLUSION}

We have implemented different image enhancement techniques and have successfully extracted the features of the affected areas. Contrast stretching is a better enhancement technique, where the dynamic range of the gray levels is increased in the image being processed. This helps in obtaining a better contrast enhanced image. We have also proposed a methodology to find out the dimensions of the affected areas such as the length, width and depth. Using our proposed methodology we can further extend the process to detect different types of defects and also severity of the defects. This system gives us to find the exact location and dimensions of the crack underground. Based on the assessment of the depth in the images using rough fuzzy set techniques, it can be decided whether the object can be mended or it needs to be replaced with a new one. This approach handles the uncertainty involved in the measuring of defects and provides a better assessment in a more efficient manner.

\section{REFERENCES}

[1] Dubois, D. and Prade, H.: Rough fuzzy sets and Fuzzy rough sets, International journal of General Systems, vol.17, no.1, (1990), pp. $191-209$.

[2]. L. A. Zadeh..: Fuzzy Sets, Information and Control, 11, (1965), pp. $338-353$.

[3] L.G. Brown: A survey of image registration techniques, ACM Computing Survey, 24 (4), (1992), pp. 352-376.

[4] M. Heath, S. Sarkar, T. Sanocki and K.W. Bowyer: A robust visual method for assessing the relative performance of edge-detection algorithms, IEEE Transactions Pattern Analysis, Mach. Intel, 19 (12) (1997), pp.1338-1359. 
[5] M. Osama and Tariq Shehab-Eldeen and, "Automated detection of surface defects in water and sewer images", Automation in Construction, 8,(1999), pp.581-588.

[6] Pawlak, Z.: Rough sets, International jour. of information and computer science, 11, (1982),pp.341 -356 .

[7] P.Maji and S.K. Pal, "Rough Set Based Generalized Fuzzy C-Means Algorithm and Quantitative Indices", IEEE Transactions on Systems, Man, and Cybernetics-Part B, Cybernetics, Vol.37, no.6, December,(2007),pp.1529-1540.

[8] S.K.Sinha and Paul W. Fieguth, "Automated detection of cracks in buried concrete image images", Automation in Construction, 15, (2006), pp.58-72.

[9] S.K.Sinha and Paul W. Fieguth, "Morphological segmentation and classification of underground image images", Machine Vision and Applications, 17(1), (2006), pp.21-31.

[10] S.K.Sinha and P.W. Fieguth, "Segmentation of buried concrete image images", Automation in Construction, 15, (2006), pp.47-57.

[11] Z. Pawlak, Rough Sets, Theoretical Aspects of Reasoning about Data. Dordrecht, the Netherlands, Kluwer, 1991.

Swarnalatha Purushotham is an Assistant Professor (Sr), in the school of computing sciences and engineering, VIT University, at Vellore, India. She is pursuing her $\mathrm{Ph} . \mathrm{D}$ degree in Intelligent Systems. She has published more than 20 papers in international journals/international conference proceedings. She is having 10 years of teaching experiences. She is a member of IACSIT, CSI, ACM, IACSIT, IEEE (WIE). Her current research interest includes Image Processing, Remote Sensing and Artificial Intelligence.

B.K Tripathy is a senior professor in the school of computing sciences and engineering, VIT University, at Vellore, India, has published more than 140 technical papers in international journals/ proceedings of international conferences/ edited book chapters of reputed publications like Springer and guided 12 students for $\mathrm{PhD}$ so far. He is having more than 30 years of teaching experience. He is a member of international professional associations like IEEE, ACM, IRSS, CSI, IMS, OITS, OMS, IACSIT, IST and is a reviewer of around 20 international journals which include World Scientific, Springer and Science Direct publications. Also, he is in the editorial board of at least 8 international journals. His current research interest includes Fuzzy sets and systems, Rough sets and knowledge engineering, Granular computing, soft computing, bag theory, list theory and social network analysis. 